interface, reducing surface tension and preventing alveolar collapse. surfactant proteins (SP)-A and D play essential antipathogenic defence and immunoregulation roles to maintain lung homeostasis. SP-D is reduced in COPD. We hypothesised lipid and protein surfactant dysregulation could contribute to pathological COPD mechanisms, this requires detailed characterisation.

Methods We conducted mass spectrometry-based multiomic (proteomic, lipidomic and metabolomic) analysis of bronchoalveolar lavage to comprehensively characterize surfactant dysregulation in a well-characterised ex-smoking mild/moderate COPD cohort $(n=31)$, with median (IQR) age of 70 (9.5) and FEV1\% predicted 73 (21), compared with healthy exsmoking volunteers $(n=20)$, with age $67.5(6.75)$ and FEV1\% predicted 100.5 (11.75). To identify the multiomic signatures of COPD, we fitted linear mixed-effects models to the data, accounting for the effects of confounding variables, such as age, gender and individual differences simultaneously. We further characterised purified alveolar macrophage gene expression differences.

Results SP-A, SP-B, and SP-D levels were lower in COPD vs control with $\log 2$ fold changes $(\mathrm{FC})$ of $-0.7\left(\mathrm{p}=1.1 \times 10^{-6}\right),-0.6$ $\left(\mathrm{p}=4.8 \times 10^{-6}\right)$ and $-0.6\left(\mathrm{p}=3.0 \times 10^{-5}\right)$, and showed positive correlations of $0.65\left(\mathrm{p}=1.5 \times 10^{-12}\right), 0.62\left(\mathrm{p}=7.7 \times 10^{-11}\right)$ and 0.43 $\left(\mathrm{p}=2.3 \times 10^{-4}\right)$ with lung function (FEV1/FVC), respectively. SP$\mathrm{C}$ was below detection limit. NAPSA and CTSH, responsible for SP-B synthesis, were lower in COPD, $\log 2 \mathrm{FC}$ of -0.1 $\left(\mathrm{p}=2.8 \times 10^{-6}\right)$ and $-0.4 \quad\left(\mathrm{p}=4.3 \times 10^{-4}\right)$, respectively. Fatty acid binding protein 4 (FABP4) and CD44, involved in surfactant regulation, were decreased in $\mathrm{COPD}, \log 2 \mathrm{FC}$ of -0.4 $\left(\mathrm{p}=8.0 \times 10^{-3}\right)$ and $-0.7 \quad\left(\mathrm{p}=1.1 \times 10^{-5}\right)$, respectively. Histamine and hypoxanthine metabolites were increased in COPD, $\log 2 \mathrm{FC}$ of $0.8 \quad\left(\mathrm{p}=2.5 \times 10^{-2}\right)$ and $1.0 \quad\left(\mathrm{p}=4.0 \times 10^{-2}\right)$, respectively, which could indicate immune-dysregulation and inflammation. Surfactant lipid levels were decreased in COPD, specifically, PC, PG, and PI, $\log 2 \mathrm{FC}$ of $-2.1 \quad\left(\mathrm{p}=2.6 \times 10^{-7}\right)$, $2.4\left(p=5.3 \times 10^{-8}\right)$ and $-1.5\left(p=9.0 \times 10^{-6}\right)$, respectively.

Conclusions We used a multiomics approach to comprehensively describe surfactant dysregulation in a well-characterised COPD cohort, with an aim to generate novel insights into key aspects of this biology within the COPD lung. Further mechanistic studies could help to understand the role this plays in pathological mechanisms and allow novel therapeutic target identification.

Please refer to page A190 for declarations of interest related to this abstract.

\section{S91 GATEWAY TO THE HIDDEN ZONE: USING PCLE TO STUDY RELATIONSHIPS BETWEEN ELASTIN REMODELLING AND SMALL AIRWAYS DISEASE IN THE COPD LUNG}

${ }^{1} \mathrm{KCW}$ Kong, ${ }^{1} \mathrm{M}$ Bennett, ${ }^{1} \mathrm{~T}$ Havelock, ${ }^{2} \mathrm{~K}$ Ostridge, ${ }^{1} \mathrm{CM}$ Spalluto, ${ }^{1} \mathrm{~T}$ Wilkinson. ${ }^{1}$ Southampton NIHR Respiratory Biomedical Research Unit, University Hospital Southampton, Southampton, UK; ${ }^{2}$ Translational Science and Experimental Medicine, Research and Early development, Respiratory and Immunology, RandD BioPharmaceuticals, Astrazeneca, Gothenburg, Sweden

\subsection{6/thorax-2021-BTSabstracts.97}

Introduction Small airways disease (SAD) is a hallmark of COPD and often precedes the development of airflow obstruction and clinically evident emphysema. SAD requires study
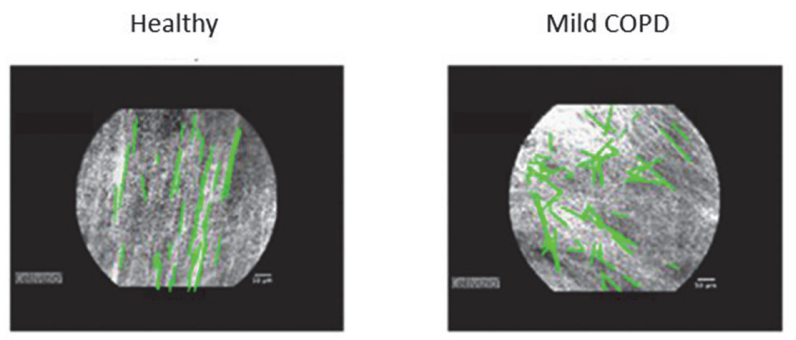

Abstract S91 Figure 1 Examples of the analysis output, with detected elastin fibres in green

however, this has been complicated by the absence of direct sampling and imaging techniques and is beyond the resolution of CT. Probe-based confocal laser endomicroscopy (pCLE) produces fluorescence images of tissue obtained in vivo during endoscopy. pCLE can image extra-cellular matrix (ECM) remodelling in the submucosa of airway walls. We developed imaging and analysis techniques and used these to describe the ECM remodelling and relationships to features of SAD and early COPD.

Methods We performed pCLE-bronchoscopy to directly visualise the airways in 8 never smokers (mean [+/- SD] age, 48 $+/-8$ ), 8 smokers with normal spirometry (mean [+/- SD] age, $55+/-5$ ) and 12 patients with COPD (mean [+/- SD] age, 61 +/- 4, mean [+/- SD] FEV1\%, 83.5\% +/- 13.5). Alveolar opening dimensions were measured, and the structural disorder caused by the remodelling process was objectively quantified and expressed as an elastin linearity score (ELS) using novel quantitative image analysis software.

Results COPD was associated with larger mean alveolar opening diameter $(350.1 \mu \mathrm{m} \pm 15.1$ vs $289.8 \mu \mathrm{m} \pm 21.6, \mathrm{p}=$ $0.000)$ and greater cross-sectional area.

There was greater disorder of airway elastin fibre alignment, even in mild COPD (mean $[ \pm$ SD] ELS, $54.9 \pm 6$ vs $44.7 \pm 9, p=0.002)$. ELS was inversely correlated with several lung function and CT imaging parameters including FEV1\% ( $\mathrm{r}=-0.477, \mathrm{p}=0.016)$ FEV1/FVC\% ( $\mathrm{r}=-0.640, \mathrm{p}$ $=0.001) \mathrm{MEF}_{25-75 \%}(\mathrm{r}=-0.649, \mathrm{p}=0.001)$, and mean expiratory to inspiratory ratio of the mean lung density (E/I MLD) $(\mathrm{r}=0.438, \mathrm{p}=0.032)$.

Conclusions This is the first pCLE study to describe airway microscopic changes related to lung function and CT indices of small airways disease in COPD. These results suggest this novel imaging analysis technique may help uncover the earliest signs of airway remodelling in COPD offering new insights into key mechanisms of disease and potential novel endpoints for development of novel disease modifying treatments for this important disease.

\section{S92 FENO NON-SUPPRESSION IDENTIFIES CORTICOSTEROID- RESISTANT TYPE-2 SIGNALING IN SEVERE ASTHMA}

${ }^{1} S$ Couillard, ${ }^{1} \mathrm{R}$ Shrimanker, ${ }^{2} \mathrm{R}$ Chaudhuri, ${ }^{3} \mathrm{AH}$ Mansur, ${ }^{4} \mathrm{LP}$ McGarvey, ${ }^{4} \mathrm{LG}$ Heaney, ${ }^{5} \mathrm{SJ}$ Fowler, ${ }^{6} \mathrm{P}$ Bradding, ${ }^{1} \mathrm{ID}$ Pavord, ${ }^{1}$ TSC Hinks. ${ }^{1}$ University of Oxford, Oxford, UK ${ }^{2}$ University of Glasgow, Glasgow, UK; ${ }^{3}$ University of Birmingham, Birmingham, UK; ${ }^{4}$ Queens University, Belfast, UK; ${ }^{5}$ University of Manchester, Manchester, UK; ${ }^{6}$ University of Leicester, Leicester, UK

\subsection{6/thorax-2021-BTSabstracts.98}

Rationale Patients with severe asthma, raised fractional exhaled nitric oxide (FeNO) and high blood eosinophil (Eos) counts are at increased risk of asthma attacks. 


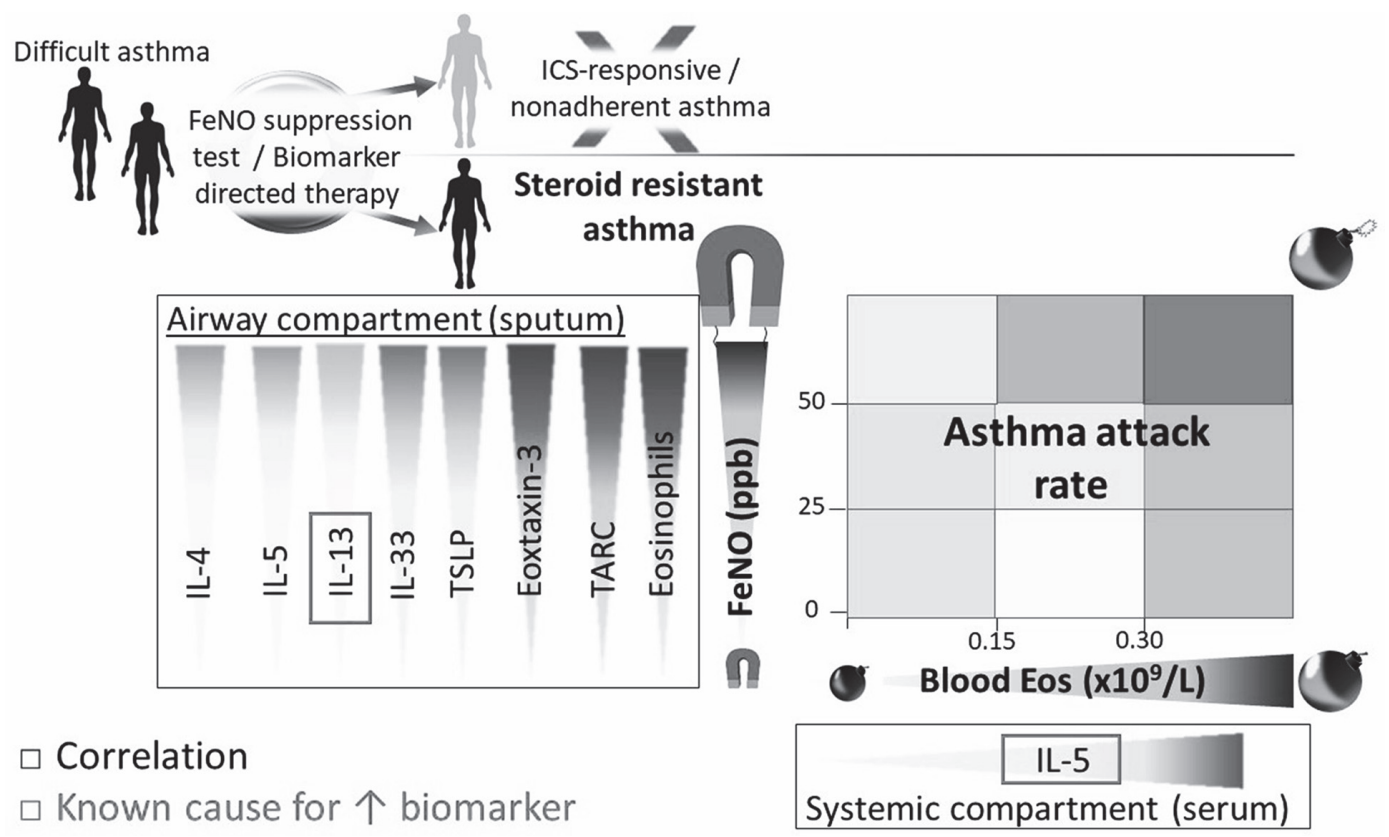

Abstract S92 Figure 1

Objective To investigate this relationship by correlating FeNO and blood Eos to inflammatory mediators in the airway and peripheral blood in severe asthma.

Methods Induced sputum Eos and 11 sputum supernatant plus 9 serum inflammatory proteins were analyzed by electrochemiluminescence or ELISA in a pooled cross-sectional analysis of patients with severe asthma and healthy controls. We recruited patients in whom we had a high degree of confidence in treatment adherence to high-dose inhaled corticosteroids (ICS) and/or systemic corticosteroids. Spearman correlations were computed between FeNO, blood Eos and sputum/serum analytes plus clinical measurements, controlling for a false discovery rate $(\mathrm{FDR})<0.05$. Significant correlations (i.e.: $\mathrm{FDR}<0.05$ ) were translated in median-fold differences across biomarker categories (FeNO: $<25,25<50, \geq 50 \mathrm{ppb}$; blood Eos: $<0.15$, $\left.0.15-<0.30, \geq 0.30 \times 10^{9} / \mathrm{L}\right)$ with Jonckheere-Terpstra tests used to assess the ordinal trends ( $p<0.05$ significant).

Results Correlation of FeNO and sputum/serum analytes in 74 patients with severe asthma showed significant correlations with sputum Eos $(r=0.51)$, interleukin (IL)-4 $(r=0.48)$, IL-5 $(r=0.47), \quad$ IL-33 $(r=0.35)$, thymic stromal lymphopoietin (TSLP: $r=0.41$ ), eotaxin-3 (CCL26: $r=0.55$ ), thymus associated regulated cytokine (TARC or CCL17: $\mathrm{r}=0.32$ ), and asthma attacks in the previous year $(r=0.25)$. Blood Eos correlated with serum IL-5 $(r=0.41)$. In effect, FeNO non-suppression was associated with higher sputum Eos (folddifference in median values, FeNO $<25$ to $\geq 50 \mathrm{ppb}$ : 17 -fold, $p$ for trend $=0.001)$, IL-4 (7.6-fold, $p=0.0006)$, IL-5 (8.9fold, $p=0.006)$, IL-33 (1.8-fold, $p=0.02)$, TSLP $(5$-fold, $p=0.002)$, eotaxin-3 (10-fold, $p=0.00003)$, TARC (3.5-fold, $p=0.005)$, and asthma attacks in the past year (3-fold, $p=0.03)$. Greater blood Eos $\left(<0.15\right.$ to $\left.\geq 0.3 \times 10^{9} / \mathrm{L}\right)$ was associated with higher serum IL-5 (1.9-fold, $p=0.04)$. Results were similar when removing patients on systemic steroids. The highest FeNO and blood Eos categories generally had greater sputum Eos, sputum/serum type-2 cytokine, chemokine and alarmin levels than healthy controls.

Conclusion Raised FeNO despite adherence to high-dose ICS identifies corticosteroid-resistant airway type-2 cytokine (IL-4,
-5), chemokine (eotaxin-3, TARC), alarmin (IL-33, TSLP) and sputum eosinophilia, while blood eosinophils correlate with systemic IL-5. These biomarkers provide complementary information on distinct immunological compartments.

Please refer to page A190 for declarations of interest related to this abstract.

\section{S93 CORRELATION OF EOTAXIN-3 GENE EXPRESSION AND OTHER IL-13-INDUCED GENES IN PATIENTS WITH ASTHMA}

${ }^{1} S$ Couillard, ${ }^{1} \mathrm{~J}$ Melhorn, ${ }^{2} \mathrm{~A}$ Singhania, ${ }^{3} \mathrm{D}$ Horowitz, ${ }^{3} \mathrm{R}$ Djukanovic, ${ }^{4} \mathrm{CH}$ Woelk, ${ }^{1}$ TSC Hinks. 'University of Oxford, Oxford, UK; ${ }^{2}$ Repare Therapeutics, Cambridge, USA; ${ }^{3}$ Janssen Research and Development, High Wycombe, UK; ${ }^{4}$ Merck Exploratory Science Center, Cambridge, USA

\subsection{6/thorax-2021-BTSabstracts.99}

Background Eotaxin-3 is an eosinophilic chemokine. We investigated how its expression in the bronchial epithelium correlates with other IL-13 signature genes in asthma.

Methods We performed a post-hoc analysis of data in patients with asthma and healthy controls (PMID\#28933920). Asthma inflammatory phenotype was determined by the induced sputum differential: eosinophilic $>3 \%$; neutrophilic $>61 \%$. Bronchial epithelial gene expression was measured using RNA microarrays on endobronchial brushes. Eotaxin-3 gene expression was compared between phenotypes by one-way ANOVA corrected for 10 comparisons. Pearson correlations $(p<0.05$ significant) were computed between eotaxin-3 and fourteen other IL-13-induced genes reported in in vitro epithelial cell studies.

Results Data from 38 asthmatics and 18 controls were included. Eotaxin-3 gene expression was highest in eosinophilic asthma (figure 1A). The correlation matrix (figure 1B) shows strong associations between eotaxin-3 gene expression and IL-13-induced-genes in patients with asthma. The 3 most correlated genes were Serpin Family B Member 10 